\title{
Generation of mesenchymal stromal cells from cord blood: evaluation of in vitro quality parameters prior to clinical use
}

\author{
Eliana Amati', Sabrina Sella ${ }^{1}$, Omar Perbellini ${ }^{1}$, Alberta Alghisi ${ }^{2}$, Martina Bernardi ${ }^{1,3}$, Katia Chieregato ${ }^{1,3}$, \\ Chiara Lievore ${ }^{2}$, Denise Peserico ${ }^{1}$, Manuela Rigno ${ }^{2}$, Anna Zilio ${ }^{4}$, Marco Ruggeri ${ }^{1}$, Francesco Rodeghiero ${ }^{3}$ \\ and Giuseppe Astori ${ }^{1 *}$
}

\begin{abstract}
Background: Increasing evidence suggests the safety and efficacy of mesenchymal stromal cells (MSC) as advanced therapy medicinal products because of their immunomodulatory properties and supportive role in hematopoiesis. Although bone marrow remains the most common source for obtaining off-the-shelf MSC, cord blood (CB) represents an alternative source, which can be collected noninvasively and without major ethical concerns. However, the low estimated frequency and inconsistency of successful isolation represent open challenges for the use of CB-derived MSC in clinical trials. This study explores whether CB may represent a suitable source of MSC for clinical use and analyzes several in vitro parameters useful to better define the quality of CB-derived MSC prior to clinical application.
\end{abstract}

Methods: CB units $(n=50)$ selected according to quality criteria (CB volume $\geq 20 \mathrm{ml}$, time from collection $\leq 24 \mathrm{~h}$ ) were cultured using a standardized procedure for CB-MSC generation. MSC were analyzed for their growth potential and secondary colony-forming capacity. Immunophenotype and multilineage differentiation potential of culture-expanded CB-MSC were assessed to verify MSC identity. The immunomodulatory activity at resting conditions and after inflammatory priming (IFN- $\gamma-1 \mathrm{~b}$ and TNF-a for 48 hours) was explored to assess the in vitro potency of CB-MSC prior to clinical application. Molecular karyotyping was used to assess the genetic stability after prolonged MSC expansion.

Results: We were able to isolate MSC colonies from $44 \%$ of the processed units. Our results do not support a role of CB volume in determining the outcome of the cultures, in terms of both isolation and proliferative capacity of CB-MSC. Particularly, we have confirmed the existence of two different CB-MSC populations named short- and long-living (SL- and LL-) CBMSC, clearly diverging in their growth capacity and secondary colony-forming efficiency. Only LL-CBMSC were able to expand consistently and to survive for longer periods in vitro, while preserving genetic stability. Therefore, they may represent interesting candidates for therapeutic applications. We have also observed that LL-CBMSC were not equally immunosuppressive, particularly after inflammatory priming and despite upregulating priming-inducible markers.

Conclusions: This work supports the use of CB as a potential MSC source for clinical applications, remaining more readily available compared to conventional sources. We have provided evidence that not all LL-CBMSC are equally immunosuppressive in an inflammatory environment, suggesting the need to include the assessment of potency among the release criteria for each CB-MSC batch intended for clinical use, at least for the treatment of immune disorders as GVHD.

\footnotetext{
* Correspondence: astori@hemato.ven.it

${ }^{1}$ Advanced Cellular Therapy Laboratory - Hematology Unit, S. Bortolo

Hospital - ULSS 6, Contra' San Francesco 41, 36100 Vicenza, Italy

Full list of author information is available at the end of the article
} 


\section{Background}

Mesenchymal stromal cells (MSC) comprise a heterogeneous population of multipotent progenitor cells used in clinic for their immunomodulatory properties and their supportive role in hematopoiesis. Three main criteria have been proposed by the International Society for Cellular Therapy (ISCT) for MSC definition: (1) adherence to plastic under standard culture conditions; (2) expression of CD105, CD73, CD90, and lack of expression of HLA-DR, together with the hematopoietic and endothelial surface markers CD14, CD45, CD34, CD11b, and CD31; (3) in vitro differentiation potential into osteocytes, chondrocytes, and adipocytes under appropriate culture conditions [1].

MSC are potent modulators of immune responses, by virtue of direct cell-cell contact and production of poorly defined soluble factors [2-4]. MSC are not constitutively inhibitory, but acquire their immunosuppressive functions following priming by inflammatory cytokines, mainly interferon gamma (IFN- $\gamma$ ) and tumor necrosis factor alpha (TNF- $\alpha)[5,6]$. The inducible MSC immunoregulatory properties are shared by MSC from bone marrow (BM) and other tissues, as well as by more differentiated fibroblasts [7].

The amenability to ex vivo expansion and the immunomodulatory activity of MSC have encouraged extensive studies paving the way for their therapeutic use, in the context of hematopoietic stem cell transplantation (HSCT) and other clinical settings [8-10]. Since 2004, the use of cryopreserved allogeneic MSC for the treatment of steroid-refractory acute graft-versus-host disease (aGvHD) has become medical practice in many countries $[11,12]$.

Although BM remains the most common source, MSC can be isolated from various human tissues [13-15]. Particularly, cord blood (CB) represents an alternative source, which can be collected noninvasively and without major clinical concerns. The network of public $\mathrm{CB}$ banks worldwide provides an easy-to-access system for the use of fresh CB units for MSC generation when they are not suitable for banking, so that CB-derived MSC can be expanded and cryopreserved in advance with enormous clinical advantages.

CB-MSC display peculiar morphological, differentiative and trophic properties [16, 17]. Some authors demonstrated a higher proliferative potential of CBMSC compared with BM- or adipose tissue-derived MSC, together with a normal karyotype after prolonged expansion [18-20]. More recently, the existence of distinct stromal $\mathrm{CB}$ populations with different performances in vitro has been postulated, on the basis of their proliferative potential, colony-forming efficiency, and telomere length [21]. Fewer studies have comprehensively addressed the immunomodulatory properties of CB-MSC, exerted on several T cell subsets and NK cells, but also through inhibition of dendritic cell function [20, 22-25].

To date, the low estimated frequency and the inconsistency of successful isolation are open challenges for the use of CB-MSC in clinical trials [26-28]. Most authors over the last years have suggested that $\mathrm{CB}$ volume and time from collection should be considered for a successful CB-MSC isolation [20, 29-31]. Recent studies have proposed efficient methods to obtain CB-MSC, avoiding strict quality selection of the starting material. These methods combined the traditional MNC separation or $\mathrm{CB}$ immunodepletion with the addition of variable supplements or coating strategies to support MSC growth [32, 33]. In this regard, the use of dexamethasone at the beginning of the culture has proven to inhibit monocyte adhesion and support CB-MSC proliferation $[20,33,34]$, without inducing changes in the subsequent differentiation potential [35].

The present study aimed at obtaining MSC from CB, by means of an isolation procedure based on the transient use of dexamethasone as medium supplement. An essential goal was to analyze several in vitro parameters useful to define the quality of CB-derived MSC in view of their clinical use. Ultimately, the immunomodulatory function during the inflammation process was assessed as a measure of their in vitro potency, with the aim to improve cell characterization.

\section{Methods}

\section{Cord blood collection}

CB was collected after maternal informed consent from the Department of Transfusion Medicine, San Bortolo Hospital (Vicenza, Italy). CB units were collected from full-term deliveries by venipuncture immediately after cord clamping and before the delivery of placenta (in utero), then stored in bags containing $30 \mathrm{ml}$ of citrate phosphate dextrose (Fresenius-Kabi, Bad Homburg vor der Höhe, Germany). Only CB units not suitable for banking with a net volume higher than $20 \mathrm{ml}$ were processed within 24 hours from the collection. Clinical information from each donor including pregnancy details and $\mathrm{CB}$ parameters was prospectively collected.

\section{CB-MSC isolation and expansion}

Mononuclear cells (MNC) were obtained by density gradient centrifugation (Lymphoprep ${ }^{\mathrm{TM}}$, Sentinel Ch. Spa, Milan, Italy) of whole $\mathrm{CB}$ diluted 1:1 with phosphatebuffered saline (D-PBS, Sigma-Aldrich, St. Louis, MO, USA). MNC were collected from the interphase, washed twice with D-PBS and plated at a density of $1-2 \times 10^{6}$ cells $/ \mathrm{cm}^{2}$ and $5-7 \times 10^{6}$ cells $/ \mathrm{ml}$ in low-glucose Dulbecco's modified Eagle's medium (DMEM) supplemented 
with $20 \%$ of fetal bovine serum (FBS) (both from Gibco, Thermo Fisher Scientific, Waltham, MA, USA), $10^{-7} \mathrm{M}$ dexamethasone (DEXA) (Hospira, Lake Forest, IL, USA), $100 \mathrm{U} / \mathrm{ml}$ penicillin and $100 \mu \mathrm{g} / \mathrm{ml}$ streptomycin (Sigma-Aldrich). Cells were then incubated at $37{ }^{\circ} \mathrm{C}$ in a humidified atmosphere containing $5 \% \mathrm{CO}_{2}$ and standard $\mathrm{O}_{2}$ concentrations. One week from initial plating, nonadherent cells were removed. Remaining cells were fed once a week and screened for colony appearance for a maximum of 4 weeks (see Additional file 1: Fig. S1). DEXA was added in the culture until the detection of MSC colonies or alternatively supplemented for only the first week of MNC culture ( $n=16$ and $n=34 \mathrm{CB}$ units, respectively; see Additional file 2: Fig. S2). MSC colonies at $80 \%$ confluence were harvested using $10 \times$ TrypLE Select (Thermo Fisher Scientific) and subcultured at a density of 4000 cells $/ \mathrm{cm}^{2}$. Standard medium was replaced twice a week and proliferation patterns were established by counting cells each week.

\section{Growth kinetics and secondary colony-forming ability of CB-MSC}

To estimate MSC growth, cells under maintenance conditions were progressively subcultured for 10-12 passages. At each subcultivation, the population doubling (PD) was calculated as follows: $\mathrm{PD}=\log _{10}(\mathrm{~N}) / \log _{10}(2)$, where $\mathrm{N}$ is the number of harvested cells/the number of initially seeded cells. The cumulative PD (cPD) was calculated adding to the PD of the passage under analysis the PDs of the previous passages.

To evaluate the secondary colony-forming ability of CB-MSC, 200 MSC collected at P1 were plated in duplicate into 100-mm diameter culture dishes (Cellstar, Grainer Bio-One GmbH, Frickenhausen, Germany) for six to seven additional passages. Standard medium was changed weekly and after 2 weeks the cells were fixed with $10 \%$ formalin, washed with deionized water and stained with May-Grunwald-Giemsa for 20 minutes. Colonies consisting of at least 30 cells were counted under an inverted light microscope (Axiovert $40 \mathrm{CFL}$, Zeiss, Oberkochen, Germany).

\section{Molecular karyotyping}

Molecular karyotyping of CB-MSC $(n=3)$ at early (P5) and late passages (P11-13) was performed through array-comparative genomic hybridization (array-CGH) with CytoChip Oligo ISCA $4 \times 180 \mathrm{~K}$ platform (BlueGnome, Cambridge, UK) and Fluorescent Labelling System (dUTP) kit (BlueGnome). High molecular weight DNA was extracted using the QIAamp DNA Mini kit (Qiagen, Hilden, Germany) according to the manufacturer's protocol. A pool of characterized genomic DNA (Human Genomic DNA Male and Female, Promega, Madison, WI, USA) was used as control DNA for all experiments. Sample and control DNA were labeled with Cy3 and Cy5 fluorophores, using random primers. Labeling mixes were combined and concentrated for hybridization. Labeled DNA was resuspended with blocking agents in hybridization buffer and applied to the CytoChip Oligo array surfaces using the gasket slides. Hybridization was performed in a rotating oven. Hybridized CytoChips were washed to remove unbound labeled DNA. A laser scanner was used to excite the hybridized fluorophores and read and store the resulting images of the hybridization. Data analysis was performed through BlueFuse Multi for Microarrays v4.0 softwarecytochip V2 algorithm (Illumina, San Diego, CA, USA). Quality control parameters for every experiment were evaluated.

\section{CB-MSC trilineage differentiation}

For osteogenic and adipogenic differentiation, CB-MSC at the end of passage 4 were seeded at a density of 4000 cells $/ \mathrm{cm}^{2}$ on cell culture coverslips (Thermo Fisher Scientific) arranged in 24-well plates (Falcon ${ }^{\circ}$, Corning, Corning, NY, USA) in the presence of standard growth medium. At $70-80 \%$ of cell confluence, the medium was replaced with specific differentiation media, then renewed every 3-4 days for 21 days. To induce adipogenic differentiation, cells were incubated using the StemPro ${ }^{\circ}$ Adipogenic Differentiation Kit (Thermo Fisher Scientific), according to the manufacturer's instructions. The presence of intracellular lipid droplets was detected by standard staining with Oil Red O (Diapath, Bergamo, Italy), according to the manufacturer's instructions. In parallel, cells were also grown using the StemPro ${ }^{\circ}$ Osteogenic Differentiation Kit (Thermo Fisher Scientific) to induce osteogenic differentiation. The presence of calcium deposits was evaluated by von Kossa staining (Sigma-Aldrich). Cells were fixed with $10 \%$ formalin for 5 minutes at room temperature, incubated with $1 \%$ silver nitrate solution for 15 minutes and exposed to ultraviolet light for 2 hours. Coverslips were rinsed with distilled water and 5\% sodium thiosulfate to remove unreacted silver. Finally, cells were counterstained with Nuclear Fast Red Solution (Sigma-Aldrich). To induce chondrogenesis, $25 \times 10^{4}$ cells were placed in a $15-\mathrm{ml}$ polypropylene tube (Falcon ${ }^{\circ}$, Corning) and washed in order to form a pelleted cellular micromass at the bottom of the tube. The cell pellet was cultured in $500 \mu$ l chondrogenic induction medium $\left(\right.$ StemPro $^{\circ}$ Chondrogenic Differentiation Kit, Thermo Fisher Scientific), following the recommendations of the manufacturer. Fresh chondrogenic medium was added every 3-4 days. After 28 days, the micromass was fixed, embedded in agar, cut with a microtome and stained with Alcian Blue (SigmaAldrich). Cells were counterstained with Nuclear Fast Red Solution. 


\section{RNA isolation and quantitative real-time polymerase chain reaction (qRT-PCR)}

Total RNA was extracted using RNeasy Plus Mini Kit (Qiagen) following the manufacturer's instructions and its quality and quantity were determined using a Nanodrop UV-VIS spectrophotometer (Thermo Fisher Scientific). First-strand cDNA were synthesized from $800 \mathrm{ng}$ of total RNA in $20 \mu \mathrm{l}$ final volume, using the iScript cDNA synthesis kit (Bio-Rad Laboratories, Hercules, CA, USA) according to the manufacturer's instructions. The mRNA expression of osteogenic markers RUNX2 and ALP, adipogeneic markers PPARG and FABP4, and chondrogenic markers SOX9 and COLXA1 was quantified by using Sso Fast evaGreen Supermix (Bio-Rad Laboratories) on the ABI 7500 Real-Time PCR System (Applied Biosystems, Thermo Fisher Scientific), according to the producer's recommendations. Primer sequences are summarized in Additional file 3: Table S1. The thermal cycling protocol involved initial denaturation at $95{ }^{\circ} \mathrm{C}$ for $30 \mathrm{sec}$ and was followed by 40 cycles of denaturation at $95{ }^{\circ} \mathrm{C}$ for $5 \mathrm{sec}$ and primer annealing and elongation for $32 \mathrm{sec}$ at $60{ }^{\circ} \mathrm{C}$, with a final melting curve analysis to test for the specificity of the product. Data acquisition and analysis were obtained by using SDS v1.4 software (Applied Biosystems, Thermo Fisher Scientific). Each gene was tested in three replicates and three independent experiments were performed. The level of each target gene was normalized to the undifferentiated control by using the $2^{-\Delta \Delta C T}$ method to quantify the relative changes in gene expression and by applying the efficiency correction represented by the equation: efficiency $=10^{(-1 / \text { slope })}-1$. TBP and YWHAZ were used as endogenous reference genes [36], provided the verification of their stability under differentiation conditions (Additional file 4: Fig. S3). PCR efficiency corrections were determined for target and reference genes by running a standard PCR curve using diluted cDNA.

\section{Immunophenotypic analysis}

Five color combinations of monoclonal antibodies (mAbs) were used to identify and characterize CB-MSC $(n=5)$ after passage 2 according to the expression of a panel of markers shown in Additional file 5: Table S2. A restricted panel was used to detect the phenotypic modifications induced on MSC by inflammatory priming (see Additional file 6: Table S3). Inflammatory priming was performed by treating CB-MSC at $80 \%$ confluence with $10 \mathrm{ng} / \mathrm{ml}$ rh-IFN- $\gamma-1 \mathrm{~b}$ (Imukin, Boehringer-Ingelheim, Ingelheim, Germany) and $15 \mathrm{ng} / \mathrm{ml}$ rh-TNF- $\alpha$ (R\&D Systems, Minneapolis, MN, USA) for 48 hours of culture, as suggested by the ISCT [37].

About $10^{5}$ cells were stained for 15 minutes at room temperature in the dark with the specific combination of
mAbs. Appropriate fluorescence-minus-one (FMO) and unstained controls were used to determine the level of unspecific binding. At least 10,000 events were acquired on a Cytomics FC500 cytometer (Beckman Coulter, Brea, CA, USA). Data were analyzed by Kaluza software 2.1 version (Beckman Coulter). Expression of individual markers was recorded as the ratio of median fluorescence intensity obtained for each marker and its negative or FMO control in the corresponding fluorescence detector (rMFI).

\section{Immunomodulation assay}

Peripheral blood mononuclear cells (PBMC) were obtained from buffy coats of healthy donors after informed consent. PBMC were isolated by density gradient centrifugation and cryopreserved until use. Thawed PBMC were suspended in RPMI 1640 (Sigma-Aldrich) supplemented with 10\% FBS, $1 \times$ L-glutamine (Sigma-Aldrich), $100 \mathrm{U} / \mathrm{ml}$ penicillin and $100 \mu \mathrm{g} / \mathrm{ml}$ streptomycin and rested overnight at $37{ }^{\circ} \mathrm{C}$ in a humidified atmosphere containing $5 \% \mathrm{CO}_{2}$ and standard $\mathrm{O}_{2}$ concentrations. Overnight resting allowed only a minimal monocyte adhesion, as shown in Additional file 7: Fig. S4. Resting and primed CB-MSC $(n=4)$, the latter stimulated for 48 hours of culture with IFN- $\gamma-1 \mathrm{~b}$ and TNF- $\alpha$, were seeded in 96-well flat-bottomed plates (Falcon ${ }^{\circ}$, Corning): $4 \times 10^{4}$ cells for the highest (1:0.2) PBMC:MSC ratio were titrated to $1 \times 10^{4}$ to achieve the lowest $(1: 0.05)$ PBMC:MSC ratio.

To measure proliferation, PBMC were stained with $5 \mu \mathrm{M}$ 5,6-carboxyfluorescein diacetate succinimidyl ester (CellTrace $^{\mathrm{Tm}}$ CFSE Cell Proliferation Kit, Invitrogen, Thermo Fisher Scientific) according to the manufacturer's instructions. CFSE-labeled cells were seeded on a MSC monolayer at different PBMC:MSC ratios: 1:0.2, 1:0.1, 1:0.05 and 1:0 (no MSC treatment). Cells were stimulated with $0.5 \mu \mathrm{g} / \mathrm{ml}$ of anti-CD3 antibody (Miltenyi Biotec, Bergisch Gladbach, Germany) and $500 \mathrm{UI} / \mathrm{ml}$ of recombinant human interleukin-2 (rh-IL-2) (Proleukin', Novartis, Basel, Switzerland) for 6 days before measuring the corresponding decrease in CFSE fluorescence by flow cytometry. For the latter, anti-human CD45-phycoerythrin-Texas Red (ECD) (J.33 clone, Beckman Coulter) mAb was used to assess proliferation on gated CD45+ cells. At least 50,000 events were acquired on a Cytomics FC500 cytometer. CFSE analysis was performed by Kaluza software and proliferation was quantified as the percentage of cells undergoing at least one cell division.

\section{Statistical analysis}

Clinical information and $\mathrm{CB}$ parameters from each donor are presented as relative frequencies or median values and their ranges for each categorical or continuous variable 
under study. The Kolmogorov-Smirnov and the Shapiro-Wilk tests were used to verify the normal distribution of each continuous variable. The differences between the continuous variables were computed by unpaired $t$ test or Mann-Whitney $U$ test as appropriate. The differences between categorical variables were computed by Fisher's exact test. Statistical comparison between resting and primed MSC (i.e., MSC treated or not with inflammatory cytokines) for each MSC batch was performed using the $t$ test for matched pairs. Proliferation data are presented as mean with SEM and statistical significance was calculated by two-way ANOVA. $P$ values $<0.05$ were considered statistically significant. Statistical analyses were performed using GraphPad Prism 5.01 software (GraphPad Software Inc., La Jolla, CA, USA).

\section{Results}

\section{CB-MSC generation}

A total of $50 \mathrm{CB}$ units with a median volume of $41 \mathrm{ml}$ (range 18-87 ml) and time after collection of $5.30 \mathrm{~h}$ (range 2-24 h) entered this study. MSC isolation was effective in $44 \%$ of processed units (22/50). Given the low frequency of MSC progenitors within CB, CB-MSC were mostly isolated as single clones, regardless of the starting volume. MSC colonies were observed at a median of 10.5 days (range 7-20) after MNC plating, while the first trypsinization occurred after a median of 13 days (range 9-22), at about 80\% confluence. Differences in either the clinical features of the donors or $\mathrm{CB}$ parameters were not globally found between successful and unsuccessful samples, as shown in Table 1.

\section{Effect of dexamethasone exposure on CB-MSC culture outgrowths}

As first approach we cultured $16 \mathrm{CB}$ units in the presence of $10^{-7} \mathrm{M}$ DEXA until the detection of MSC growing colonies [34]. CB-MSC clones were isolated from $37.5 \% \mathrm{CB}$ units $(6 / 16)$. Colonies were detected at a median of 12.5 days from initial plating (range 8-20) and harvested after a median of 13.5 days (range 13-22). All samples except one reached at least five passages.

To assess whether a lower exposure to DEXA could improve CB-MSC isolation and proliferation capability, a second series of $\mathrm{CB}$ units $(n=34)$ was subjected to DEXA supplementation for the first week of MNC culture only. In this condition, MSC isolation was successful in $47.1 \%$ units $(16 / 34)$, with a median detection and harvest time of 10 (range 7-15) and 12 days (range 9-15), respectively. All samples were capable to reach at least five passages.

The withdrawal of DEXA after the first week of MNC culture did not significantly modify either the efficiency of CB-MSC isolation ( $p=0.5253$, Fig. 1a) or the $\mathrm{cPD}$ at P5 ( $p=0.0867$, Fig. 1b).

\section{CB-MSC growth characteristics}

The isolated CB-MSC displayed initially a small spindleshape morphology and a high degree of heterogeneity, mainly due to the contamination by osteoclast-like cells and non-proliferating fibroblast-like cells. These contaminating cells that were strongly adhered to the bottom of the flasks were eliminated by P2 passage (Fig. 2a-c).

Differences in the proliferative capacity and exhaustion passage were observed between MSC from different units. Overall, $1 / 3$ of CB-derived MSC were able to

Table 1 Comparison between donor characteristics and successful CB-MSC isolation

\begin{tabular}{|c|c|c|c|c|}
\hline & All samples $(n=50)$ & MSC-positive isolation $(n=22)$ & MSC-negative isolation $(n=28)$ & $p$ value \\
\hline Median time after delivery, hours (range) & $5(2-24)$ & $5(2-24)$ & $7(2-24)$ & $0.799^{\circ}$ \\
\hline Median TNC $\times 106$ (range) & $696(276-1700)$ & $675(383-1290)$ & $700(276-1700)$ & $0.662^{\circ}$ \\
\hline Median MNC $\times 106$ (range) & $191(14-615)$ & $200(85-615)$ & $191(14--62)$ & $0.703^{\circ}$ \\
\hline CB median volume, $\mathrm{ml}$ (range) & $41(18-87)$ & $43(22-87)$ & $40(18-78)$ & $0.323^{\S}$ \\
\hline Median gestational time, days (range) & $273(259-292)$ & $273(264-292)$ & $275(259--92)$ & $0.288^{\circ}$ \\
\hline Median mother age, years (range) & $35(26-45)$ & $34(29-41)$ & $35(26-45)$ & $0.799^{\S}$ \\
\hline Pluriparity & $27(54 \%)$ & $11(50 \%)$ & $16(57.1 \%)$ & $0.198^{*}$ \\
\hline Male babies & $27(54 \%)$ & $14(63.6 \%)$ & $13(46.4 \%)$ & $0.264^{*}$ \\
\hline Median baby weight, grams (range) & $3390(2430-4460)$ & $3335(2700-4460)$ & $3405(2430--090)$ & $0.674^{\S}$ \\
\hline Mater/baby blood group match & $26(52 \%)$ & $15(68.2 \%)$ & $11(39.3 \%)$ & $0.052^{*}$ \\
\hline Cesarean birth & $14 / 48(29.2 \%)$ & $7 / 20(35 \%)$ & $7 / 28(25 \%)$ & $0.528^{*}$ \\
\hline
\end{tabular}

Abbreviations: MSC mesenchymal stromal cells, TNC total nucleated cells, MNC mononuclear cells, CB cord blood Statistical tests:

${ }^{\circ}$ Mann-Whitney $U$ test

sUnpaired $t$ test

*The differences between the categorical variables were computed by the Fisher exact test 


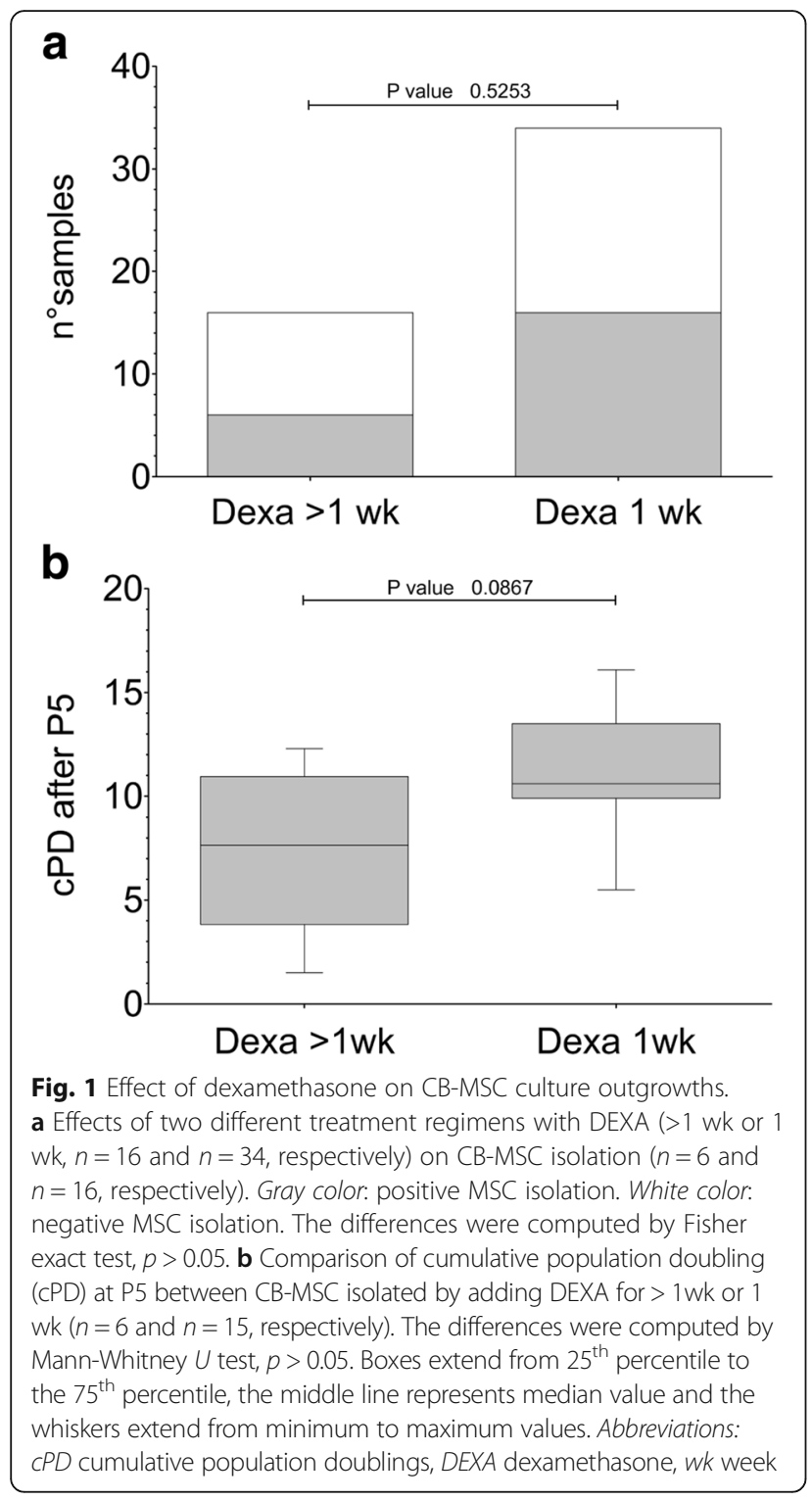

expand for more than nine passages. By evaluating the long-term proliferative potential at least two growth kinetics patterns were recognized. We distinguished short- and long-living (SL- and LL-) CB-MSC based on their lower or higher $\mathrm{CPD}$, respectively $(\mathrm{cPD}$ cutoff $=20$ at p9). LL-CBMSC displayed a constant greater growth and longevity than SL-CB-MSC (Fig. 2d). Moreover, by comparing the $\mathrm{CPD}$ at each passage, significant differences in the proliferative capacity were revealed by passage 5 (Fig. 2e).

Since the discrimination between SL- and LL-CBMSC based on the CPD could only be done retrospectively, we sought to identify an earlier distinctive marker, possibly of clinical utility for the choice of the batches of CBMSC suitable for large-scale expansion and clinical use. As already demonstrated, the heterogeneous proliferative potential reflected differences in the self-renewal capacity $[21,38]$. By assessing the secondary colony-forming capability of the two populations, we found that LLCBMSC retained greater secondary colony-forming ability compared to SL-CBMSC. Conversely, SL-CBMSC failed to self-renew after a few passages then lost the growth capacity earlier (Fig. 2f-g). Significant differences were specifically observed at passage 4 , albeit on a limited number of samples (Fig. 2h).

We next addressed the role of donor characteristics and CB parameters (listed in Table 1) as discriminating markers between LL- and SL-CBMSC. Quite surprisingly, we found that the median of $\mathrm{CB}$ volumes of units giving rise to SL-CBMSC was significantly higher (51 ml, range 22-87) with respect to the volume of CB units giving rise to LL-CBMSC (31 ml, range 27-42) ( $p=0.0388, n=16$ and $n=5$, respectively, Fig. $2 \mathrm{i}$ ).

Finally, in order to test the genetic stability of CBMSC after prolonged expansion, three LL-CBMSC batches at early (P5) and late passages (P11-13) were tested for their genomic assets through array-CGH analysis. Results revealed that expanded CB-MSC did not show unbalanced chromosomal rearrangements (deletion or duplication), excluding copy number variation constitutionally present (see Additional file 8: Fig. S5).

\section{Multilineage differentiation}

To investigate the in vitro differentiation potential of CB-MSC from various LL donors, cells at P4 were induced to differentiate down the osteogenic, adipogenic and chondrogenic lineages, by using defined media components and culture conditions (Fig. 3a-f). All CB-MSC $(n=5)$ demonstrated osteogenic differentiation after 3 weeks of induction. By contrast, we observed poor adipogenic potential (1/5 samples) as revealed by Oil Red O staining. When cultured under chondrogenic conditions, cartilage-like cells with lacunae and a large amount of cartilage extracellular matrix were observed in sections of pellets from all samples. Parallel experiments on SL-CBMSC confirmed the absence of dissimilarities compared to LL-CBMSC in regard to osteogenic and adipogenic multilineage differentiation (Additional file 9: Fig. S6), while chondrogenic potential was not assessed due to the difficulty to obtain a sufficient number of SL cells for the assay.

To confirm multilineage differentiation at a molecular level, the transcript levels of both early- and late-stage markers of adipogenesis, osteogenesis, and chondrogenesis were determined by means of qRT-PCR in LL-CBMSC. Results from three independent experiments confirmed, even with variability between MSC donors, significant upregulation of all mRNA transcripts involved in chondrogenic and osteogenic MSC differentiation $(p=0.0039$ for SOX9, RUNX2, and ALP; $p=0.0078$ for COLXA1), while 

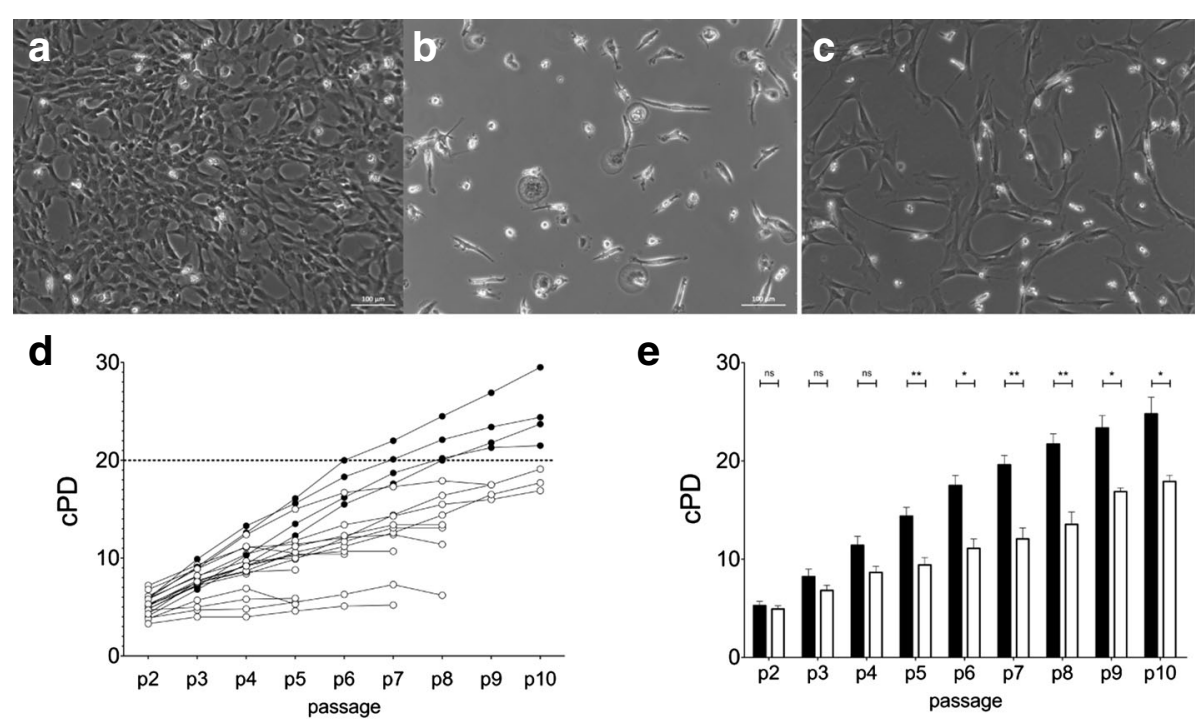

e

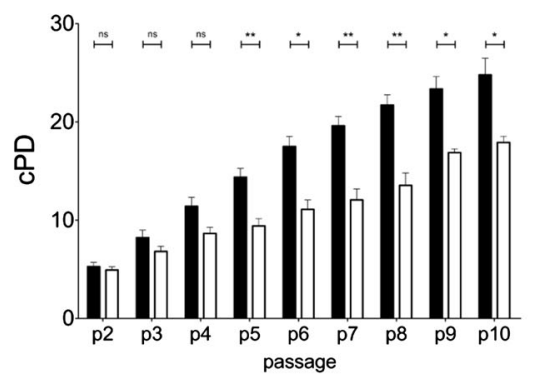

f

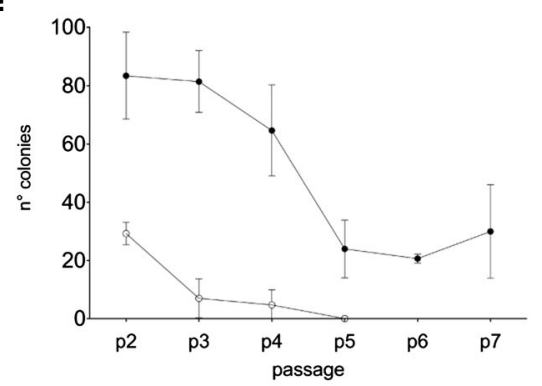

h

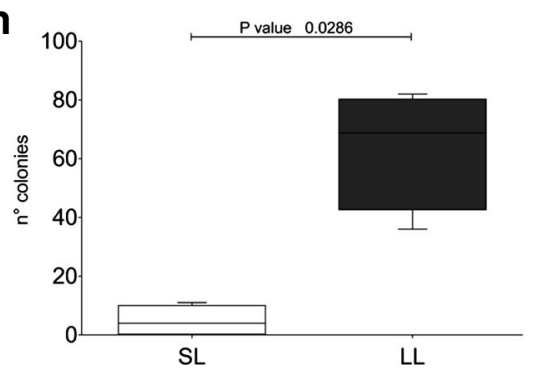

g

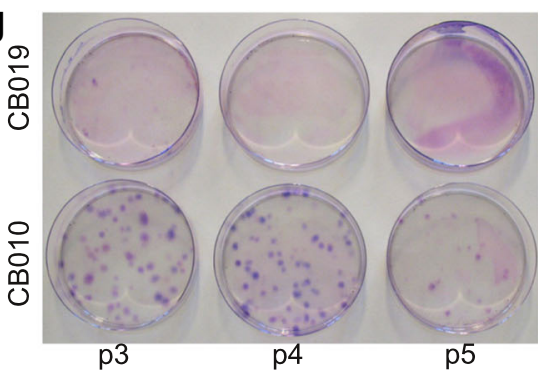

i

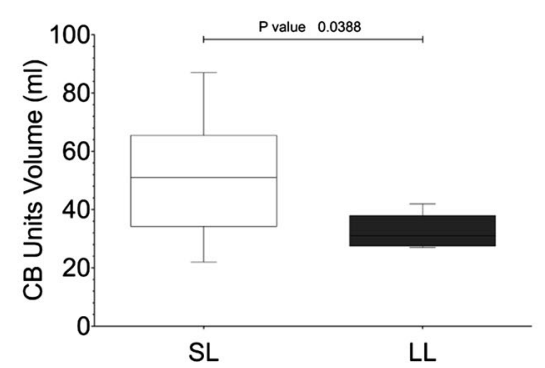

Fig. 2 Morphology and growth characteristics of CB-MSC. a Colony of CB-MSC 10 days after initial seeding (passage 0). b Non-proliferative fibroblast-like cells and osteoclast-like cells, the latter with very large cytoplasm and occasional multiple nuclei (passage 0). c Morphology of CB-MSC at passage P1. Scale bars: $100 \mu \mathrm{M}$. d Growth patterns of CB-MSC grouped by similar CPD (cPD cutoff $=20$ at P9). Black circles: LL-CBMSC; white circles: SLCBMSC. e Comparison of CPD between LL- (black bars) and SL- (white bars) CBMSC at each passage; the differences were computed by Mann-Whitney $U$ test, ${ }^{*} p<0.05,{ }^{* *} p<0.01,{ }^{* * *} p<0.001$; data are presented as mean with SEM. $\mathbf{f}$ Secondary colony formation of LL-CBMSC (black circles) and SL-CBMSC (white circles) at defined passages. $\mathbf{g}$ Colonies formed after plating $200 \mathrm{MSC}$ in 100-mm culture dishes are shown from one representative LL-and one SL-CBSMC (CB010 and CB019, respectively). $\mathbf{h}$ Secondary colony formation of LL-CBMSC (black boxes) and SL-CBMSC (white boxes) at P4. The differences were computed by Mann-Whitney $U$ test, $p<0.05$. Boxes extend from $25^{\text {th }}$ percentile to the $75^{\text {th }}$ percentile, the middle line represents median value and the whiskers extend from minimum to maximum values. i Comparison between CB volumes between LL-CBMSC and SL-CBMSC ( $n=5$ and $n=16$, respectively); the differences were computed by Mann-Whitney $U$ test, $p<0.05$. Abbreviations: LL-CBMSC long-living CBMSC, SL-CBMSC short-living CBMSC, NS not significant

the absence of significant differences for the adipogenic markers PPARG and FABP4 ( $p=0.0547)$ (Fig. 3i-j).

\section{Immunophenotypic analysis}

Immunophenotypic characterization was performed by flow cytometry in agreement with ISCT criteria. Relevant
MSC-related and pericyte markers were investigated based on current literature [39]. Culture-expanded LLCBMSC $(n=5)$ strongly expressed the MSC markers CD90, CD105, CD44, CD13, and HLA-ABC, while they were negative for the hematopoietic markers CD31, CD34, CD45, and for HLA-DR. Additional markers 

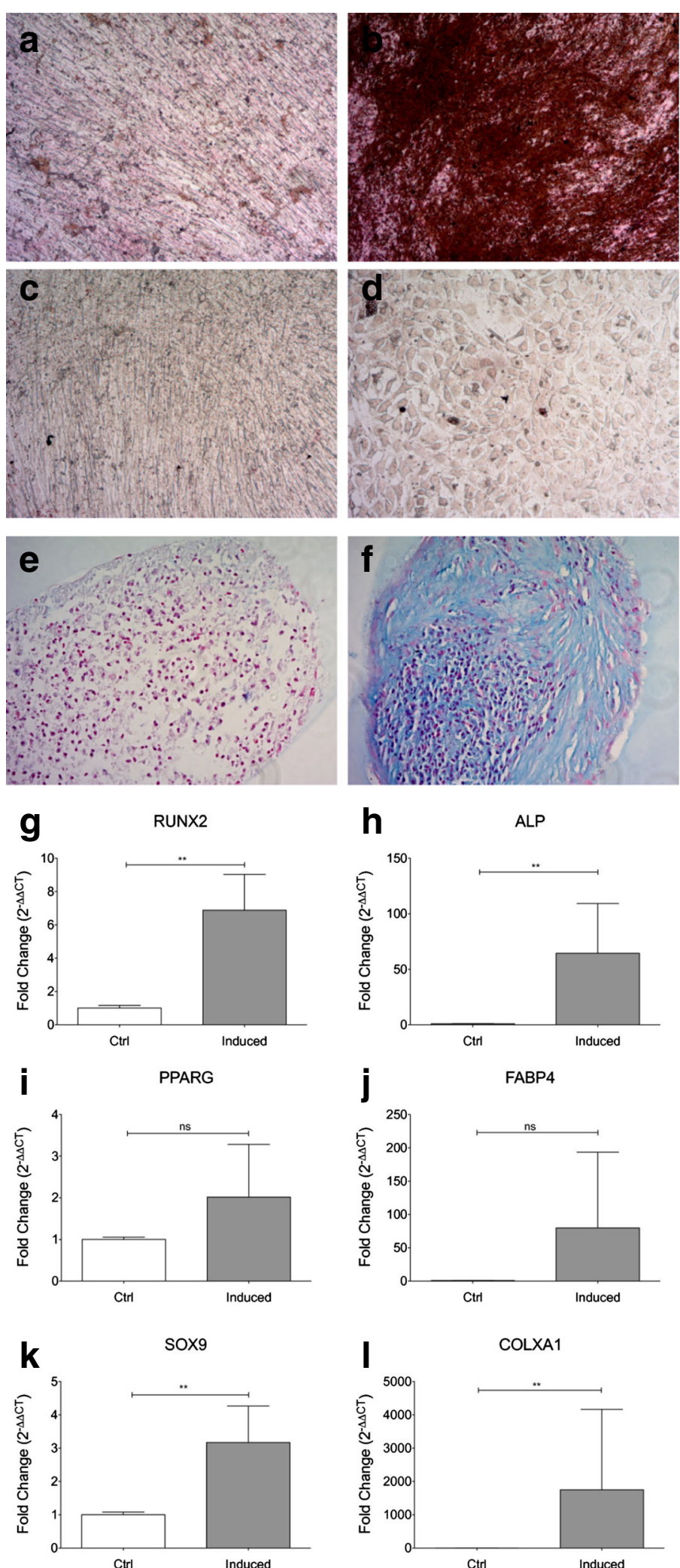

Fig. 3 (See legend on next page.) 
(See figure on previous page.)

Fig. 3 Multilineage differentiation of CB-MSC. Multilineage ability was determined in P4 LL-CBMSC. a-f Panels display cells which have been induced to differentiate in vitro toward osteogenic (a-b), adipogenic ( $\mathbf{c}-\mathbf{d})$, and chondrogenic (e-f) lineages. Osteogenic and adipogenic differentiation were assessed after 21 days of induction using von Kossa and Oil Red O staining, respectively; $\times 10$ magnification. Chondrogenesis was evaluated by Alcian Blue staining at day 28 of induction; cells were counterstained with Nuclear Fast Red solution; $\times 20$ magnification. For each staining, undifferentiated controls are also displayed on the left (panels a-c-e). $\mathbf{g}$ Quantitative RT-PCR analysis of osteogenic markers RUNX2 and ALP ( $\mathbf{g}-\mathbf{h}$ ), adipogenic markers PPARG and FABP4 $(\mathbf{i}-\mathbf{j})$, and chondrogenic markers SOX9 and COLXA1 (k-1) in cells cultured under the respective lineage induction conditions. Results are presented as the fold change in mRNA expression in respect to TBP as representative reference gene and to the undifferentiated control. The mean values from three independent experiments done in triplicate are shown. The differences were computed by paired $t$ test or Wilcoxon matched pairs test as appropriate, $p$ values: ${ }^{* *} p<0.01$. Abbreviations: NS not significant

searched for on the MSC surface showed variable expression, such as the perivascular antigens PDGFR $\beta$, CD146, and NG2 (Fig. 4a). As already reported by other authors, CB-MSC were found negative for CD271 $[20,40]$. None of the investigated markers was found differentially expressed on the surface of LLcompared to SL-CBMSC (Additional file 10: Fig. S7).

We then evaluated the modifications of MSC immunophenotype after treatment with IFN- $\gamma-1 b$ and TNF- $\alpha$ for 48 hours, corresponding to induction of immunosuppressive function in MSC [5]. As previously demonstrated, the expression of HLA-ABC, HLA-DR, CD54 (ICAM-1), and CD106 (VCAM-1) was modulated in the presence of inflammatory priming [40]. Particularly, significant upregulation was observed for CD54 (low-negative at resting conditions) and HLA-ABC (high-positive at resting conditions) ( $p=0.004$ and $p<0.001$, respectively, Fig. 4 c-d). Upregulation of CD106 (low-negative at resting conditions) did not reach significance, while the expression of HLA-DR (negative in resting MSC) was almost unchanged (Fig. 4b-e).

\section{Immunosuppressive properties of CB-MSC}

MSC are known for their remarkable ability to suppress the proliferation of several immune cell types [2]. We tested the immunosuppressive properties of CB-MSC (specifically LL-CBMSC) by assessing their capacity to modulate the proliferative response of CFSE-labeled PBMC upon stimulation with anti-CD3 and rh-IL-2. MSC batches $(n=4)$ at P5-P6 were analyzed, provided with additional experiments that there were no differences in the inhibitory potential with passaging (e.g., from P2 to P6) on both resting and primed MSC (Additional file 7: Fig. S4). Flow cytometry analysis of CFSE dilution on CD45+ cells showed that proliferation of activated PBMC was generally suppressed by MSC in a dose-dependent manner (Fig. 5a-b). Nevertheless, significant differences in the inhibitory potential were revealed between individual MSC batches, particularly after IFN- $\gamma-1 \mathrm{~b}$ and TNF- $\alpha$ priming (Fig. 5b). We thus expressed the MSC inhibitory potential in terms of proliferation ratio, as the ratio between the percentage of $\mathrm{CD} 45+$ proliferation at primed and resting conditions. In most cases, the proliferation ratio increased inversely with MSC dose. For only one CB-MSC batch, a proliferation ratio directly increasing with MSC dose was observed, suggesting the lack of inhibition by inflammatory-primed MSC on PBMC proliferation (Fig. 5c). In this case, the proliferation ratio was found significantly greater with respect to other batches, specifically at 1:0.2 and 1:0.1 PBMC:MSC ratio $(p<0.001$ and $p<0.01$, respectively, Fig. 5 c).

\section{Discussion}

Obtaining definitive data on the effectiveness of MSC in the clinic is hampered by the lack of standardized protocols used to prepare large-scale MSC and of useful tests to compare their potency. Particularly, differences in donor source, culture methods, and expansion levels are critical in determining MSC functionality $[41,42]$.

Our study investigated whether $\mathrm{CB}$ may represent a suitable source of MSC for cell-based therapeutic strategies. Furthermore, the biological and functional properties of CB-derived MSC were assessed in view of a more effective and safer clinical use. By applying quality criteria for an optimal CB-MSC isolation (CB volume $\geq 20 \mathrm{ml}$, time from collection $\leq 24 \mathrm{~h}$ ), we were able to isolate MSC colonies from $44 \%$ of processed units. We next evaluated whether isolation was influenced by any clinical features of the donors or $\mathrm{CB}$ parameters, but we found no correlation between the analyzed parameters and the rate of success in isolating CB-MSC. Other studies reported isolation yields ranging from fewer than $10 \%$ to $90 \%$, revealing a lack of consensus in the methodological approaches and selection criteria for CB units [20, 21, 29, 30, 43]. By using DEXA $\left(10^{-7} \mathrm{M}\right)$ as medium supplement in addition to $20 \% \mathrm{FBS}$ for 1 week, Zhang et al. achieved a $90 \%$ rate of success in isolating CB-MSC when the volume was $\geq 90 \mathrm{ml}$ and the time to processing $\leq 2 \mathrm{~h}[20]$. By applying the same criteria, Pievani et al. were able to obtain MSC from $40 \%$ of processed units only [35].

DEXA was found to inhibit monocyte adhesion, thus it is conceivable a role of the steroid in supporting the proliferation of CB-MSC progenitors at the expense of other contaminants which can adhere on culture plates. Therefore, its supplementation was applied also in our study with the aim to promote the adhesion of the rare 


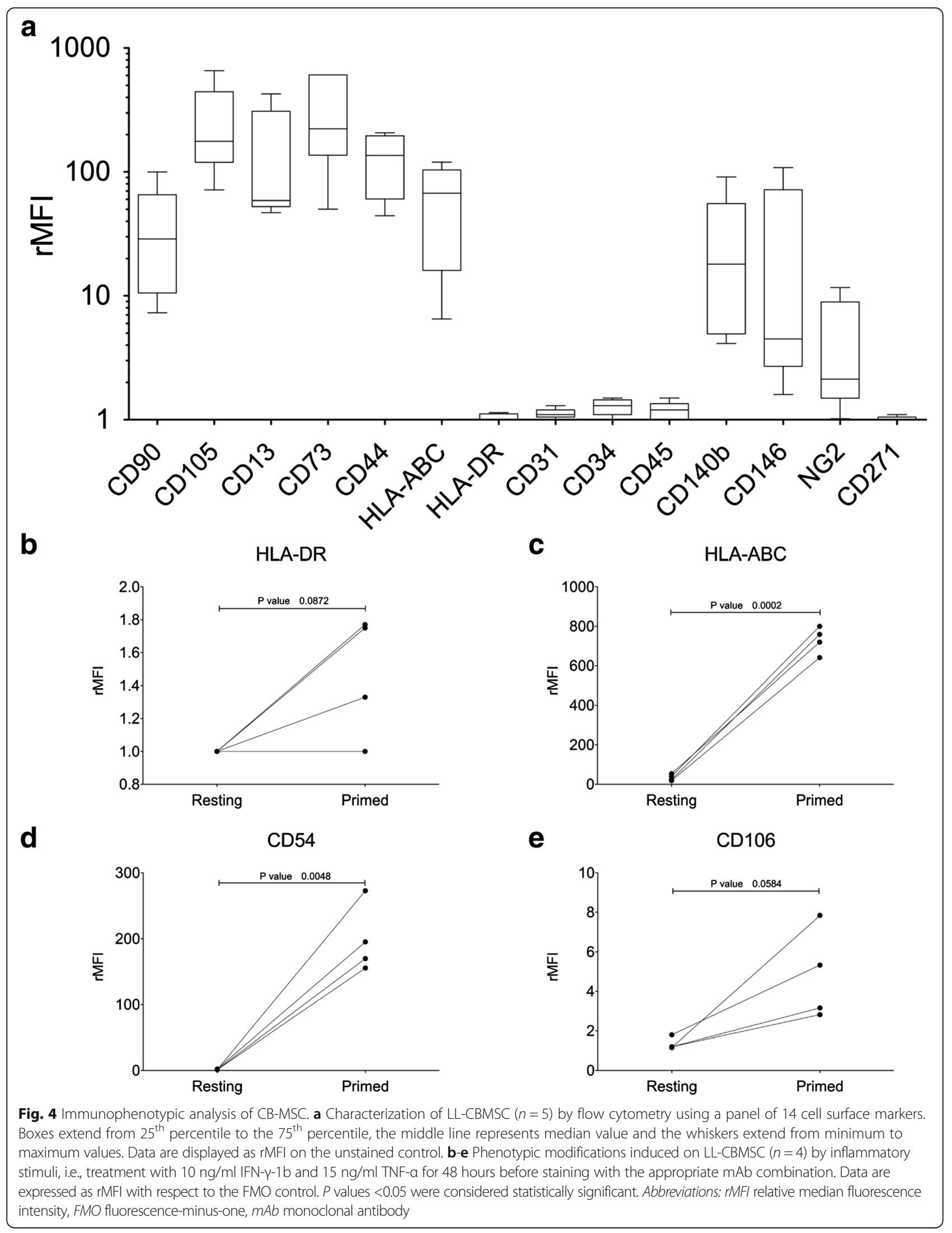




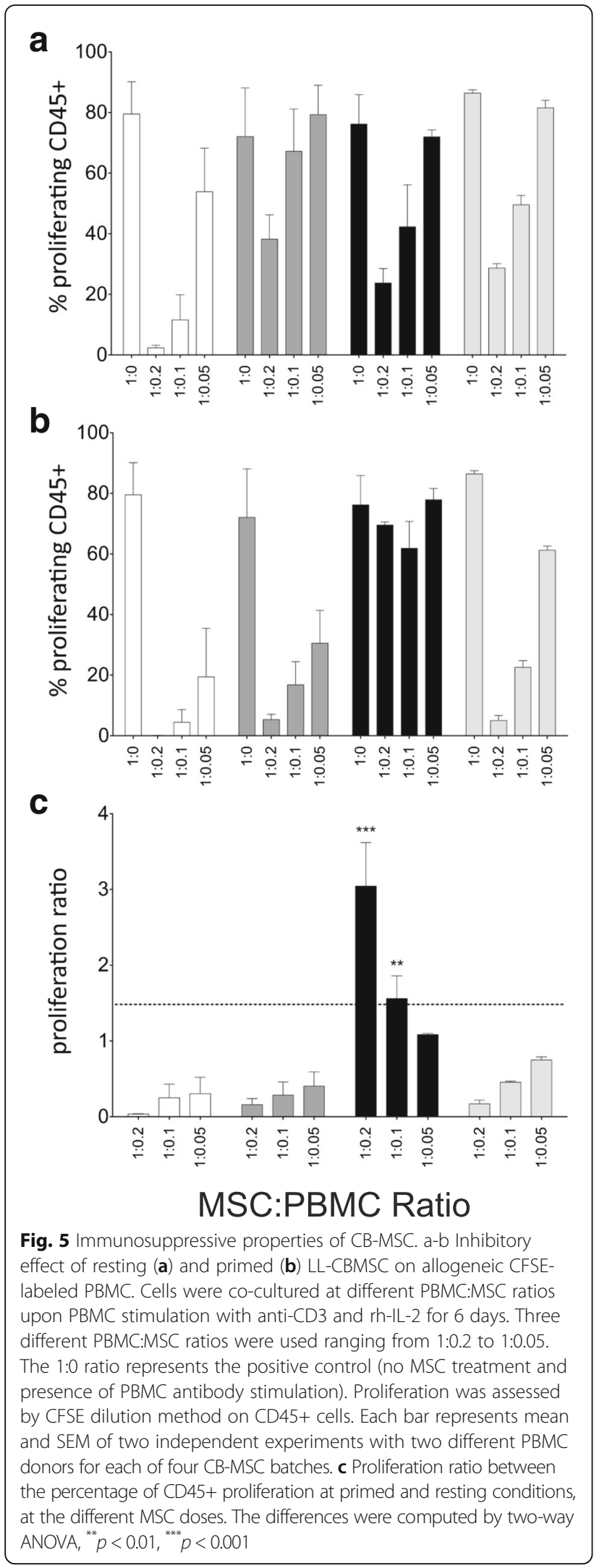

CB-MSC progenitors to the plastic. Moreover, Zhang and co-workers found no benefit from using alternative isolation methods or culture conditions, such as immunodepletion before MNC plating, addition of growth factors to the standard MSC medium, culture in hypoxia, or coating strategies [20]. Even though the use of DEXA has been described in the generation of CBMSC [20, 34, 35], its dosage varied between investigators. In the first isolation protocol published by Kögler et al. [34], DEXA $\left(10^{-7} \mathrm{M}\right)$ was added until the detection of MSC growing colonies, while the expansion was performed in the absence or at a low concentration of the supplement. In the present study, the choice to evaluate two treatment regimens with DEXA was of particular significance in order to define a standardized protocol for generating CB-MSC for clinical use. Our results showed that the extent of DEXA supplementation did not significantly affect CB culture outcomes, in regard to both isolation efficiency and long-term proliferation, even if there seemed to be better outcomes in the group of units treated with DEXA for only 1 week. Given that culture conditions may have remarkable effects on the functionality of MSC [33, 44], the addition of uncommon supplements to standard MSC medium, like hormones, should be avoided or minimized, at least when the purpose is to produce MSC with unaltered stemness properties for clinical use. It should be therefore more appropriate and equally effective keeping cell exposure to DEXA to the minimum required for an effective CB-MSC isolation.

As discussed, there are some drawbacks linked to the successful isolation and expansion of CB-MSC, mainly due to the low frequency of MSC clones in particular when compared with umbilical cord (UC), a rich source of high-proliferative MSC characterized by isolation yields of $100 \%$ [45]. To the other hand, UC is a heterogeneous tissue whose processing is time-consuming and labor-intensive in respect to the easier manipulation of CB. Moreover, public CB banks provide an easy-toaccess system for using freshly donated $\mathrm{CB}$ units for MSC generation, while a similar collection network system does not exist for UC or other fetal sources like placenta. The present study has also revealed that the role of volume as selection criterion for $\mathrm{CB}$ units processed for CB-MSC isolation should be re-considered because of affecting neither the rate of successful MSC isolation nor the growth potential. This finding would allow to use even CB units of low volume for MSC isolation.

An important outcome of the present study was to analyze several in vitro parameters that may help to better define the "quality" of CB-derived MSC prior to their clinical application. The evaluation of the proliferation capacity as first indicator of MSC potential allowed us to 
corroborate the findings from Barilani and co-authors [21], who demonstrated the existence of at least two stromal populations within $\mathrm{CB}$, one long-living (LLCBMSC) and the other short-living (SL-CBMSC) on the basis of their proliferative ability, secondary colonyforming efficiency and most importantly, telomere length. The same authors found LL-CBMSC mostly indiscernible from the SL counterpart in regard to ISCT criteria [32]. We confirmed that the two MSC populations clearly diverge in their growth capacity and secondary colony-forming efficiency, thus suggesting a potential role of these in vitro parameters as indicators of CB-MSC longevity. On one hand, the possibility of cryopreserving low-passage CB-MSC batches in advance in respect to the clinical needs should allow to monitor cell growth until the established cPD cutoff, thus rendering the retrospective discrimination between LL- and SL-CBMSC clinically useful. On the other hand, the evaluation of secondary colony formation may be a promising and likely early parameter of longevity.

Our data confirmed the absence of major dissimilarities concerning immunophenotype and multilineage differentiation, fitting both populations the minimal panel proposed for MSC definition [1]. It is conceivable that LL-CBMSC represent better candidates for obtaining clinically relevant numbers of MSC for therapeutic applications, due to the higher ex vivo expansion and the ability to survive for longer periods in vitro. Therefore, the assessment of their genetic stability after prolonged culture represents an important release criterion for a safe clinical use, even if overly expansion should be avoided too [46, 47].

Unlike MSC from other sources, is widely reported the relatively low adipogenesis ability of CB-MSC under standard induction protocols [48-50], most likely due to their more primordial, fetal origin in respect to other MSC sources [51]. On the other hand, it is well documented that there is more propensity toward chondrogenesis and osteogenesis in vitro under appropriate culture conditions and to some extent in vivo [20, 35, 52, 53]. Sacchetti and co-workers [53] have recently demonstrated a unique capacity of CB-derived stromal cells to form cartilage in vivo spontaneously, in addition to an osteogenic capacity. Therefore, they support the presence within $\mathrm{CB}$ of chondro-osteoprogenitors rather than multipotent MSC, whose origin, however, remains to be elucidated. Our data confirmed an impaired adipogenic potential of CB-MSC, revealed by a clear absence of cells forming lipid droplets and confirmed at a molecular level by the absence of significant mRNA induction of PPARG and FABP4, the former representing a crucial player during the transcriptional cascade leading to adipogenic differentiation, the latter being its direct target. More in general, our molecular analysis highlighted a wide variability in the differentiation potential between individual samples. This finding may fit the increasing evidence of the existence of distinct CB-derived stromal progenitors, possibly of different developmental origin and related plasticity [53-56]. In this regard, unrestricted somatic stromal cells (USSC) and cord blood-derived stromal cells (CB-MSC) were originally defined according to the expression of the adipogenic inhibitor delta-like 1 (DLK-1), a specific marker of USSC correlating with a lack of adipogenic differentiation ability and a higher proliferative potential compared to the more differentiated and less proliferative CB-MSC [54]. Even if in our experience a lack of adipogenic potential was common to both LL- and SL-CBMSC, it remains unclear whether the more immature USSC match our LL-CBMSC.

The ultimate aim of the present work was to investigate the immunosuppressive activity of both resting and inflammatory-primed CB-MSC, in order to complete their functional characterization prior to clinical application. Despite the recent suggestions provided by ISCT $[37,42,57]$, a universally accepted in vitro method to assess MSC immunosuppression does not exist [58, 59]. We used unselected and opportunely stimulated PBMC because more closely mimic the in vivo inflammatory environment to which MSC are exposed on patient administration. Our results showed that CB-MSC inhibited PBMC proliferation with different efficacy, particularly after treatment with exogenous IFN- $\gamma-1 \mathrm{~b}$ and TNF- $\alpha$. The lack of efficacy of one batch was found despite the flow cytometry upregulation of priming-inducible markers. Moreover, it was confirmed repeatedly on different PBMC donors. In this situation, a trophic effect mediated by primed CB-MSC was conceivable on PBMC proliferation. Additional studies would be warranted to determine how correlating this observation with other immunomodulatory markers, for adequately predicting the in vivo potency of CB-MSC. von Bahr and co-authors [60] reported no evidence of correlation between the in vitro inhibitory potential of MSC and in vivo clinical response in patients with aGvHD, suggesting that the in vivo efficacy of MSC not only depends on the intrinsic properties of the MSC preparation but also on the product-host interaction. A detailed immunomonitoring of patients as well as an in-depth characterization of MSC would therefore be helpful to correlate the in vitro measure of the potency with clinical outcomes [61].

\section{Conclusions}

In summary, the present work shows that $\mathrm{CB}$ may be a practical source of MSC for clinical applications. CB remains much more readily available and devoid of major ethical concerns in respect to other conventional MSC sources. To the best of our knowledge, we have 
provided evidence that not all LL-CBMSC are equally immunosuppressive in an inflammatory environment, in support of the need to include the assessment of in vitro potency among the release criteria for each CB-MSC batch intended for clinical use, at least for the treatment of immune disorders such as GvHD.

\section{Additional files}

Additional file 1: Figure S1. Schematic protocol for CB-MSC generation. Success or failure in isolation of CB-MSC was assessed by the appearance of colonies to a maximum of 4 weeks from MNC plating. Abbreviations: MNC mononuclear cells. (DOCX $104 \mathrm{~kb}$ )

Additional file 2: Figure S2. Dexamethasone scheduling in MNC culture. The supplement was added in standard medium until the detection of MSC colonies ( $n=16$ CB units) or alternatively added for the first week of MNC culture only ( $n=34$ ). (DOCX $120 \mathrm{~kb}$ )

Additional file 3: Table S1. Genes and primer sequences used for quantitative real-time PCR (RT-PCR). (DOCX $13 \mathrm{~kb}$ )

Additional file 4: Figure S3. Stability of endogenous reference genes (TBP and YWHAZ) under differentiation conditions. Quantitative RT-PCR analysis of TBP normalized with respect to YWHAZ (A-C) and vice versa (D-F) under osteogenic (A and D), chondrogenic (B and $E$ ) and adipogenic ( $\mathrm{C}$ and $\mathrm{F}$ ) differentiation conditions. Results are presented as the fold change in mRNA expression obtained by using the $2^{-\Delta \Delta C T}$ method, applying the correction efficiency for each gene. The mean values from three independent experiments done in triplicate are shown. The differences were computed by Wilcoxon matched pairs test, $p>0.05$. Abbreviations: NS not significant. (DOCX $526 \mathrm{~kb}$ )

Additional file 5: Table S2. List of antibodies used for immunophenotypic analysis. (DOCX $14 \mathrm{~kb}$ )

Additional file 6: Table S3. Five-color mAb combinations used for immunophenotypic analysis of inflammatory MSC priming. (DOCX $14 \mathrm{~kb}$ )

Additional file 7: Figure S4. Immunomodulation assay. Flow cytometry analysis of thawed PBMC before (A) and after (B) overnight resting. The percentage of monocytes of one representative sample is shown. (C) Proliferation ratio between the percentage of CD45+ proliferation at primed and resting conditions, at the different MSC passages. Results from two independent experiments against two different PBMC lots are shown. (DOCX 637 kb)

Additional file 8: Figure S5. Representative array-CGH profiles of the whole genome of one representative LL-CBMSC sample at P5 (lower panel) and P11 (upper panel) (DOCX $107 \mathrm{~kb}$ )

Additional file 9: Figure S6. SL-CBMSC multilineage differentiation. Panels display cells which have been induced to differentiate in vitro toward osteogenic (A-B) and adipogenic (C-D) lineages. Osteogenic and adipogenic differentiation were assessed after 21 days of induction using von Kossa and Oil Red $O$ staining, respectively; $\times 10$ magnification. For each staining, undifferentiated controls are also displayed on the left (panels A-C). One representative sample is shown. (DOCX $2.42 \mathrm{mb}$ )

Additional file 10: Figure S7. SL-CBMSC immunophenotypic analysis. Characterization of SL-MSC $(n=6)$ by flow cytometry using a panel of 14 cell surface markers. Boxes extend from $25^{\text {th }}$ percentile to the $75^{\text {th }}$ percentile, the middle line represents median value and the whiskers extend from minimum to maximum values. Data are displayed as rMFI on the unstained control. (DOCX $104 \mathrm{~kb})$

\section{Abbreviations}

aGvHD: acute graft-versus-host disease; BM: bone marrow; CB: cord blood; CFSE: carboxyfluorescein diacetate succinimidyl ester; CGH: comparative genomic hybridization; CPD: cumulative population doublings; DEXA: dexamethasone; DMEM: Dulbecco's modified Eagle's medium; ECD: phycoerythrin-Texas Red; FBS: fetal bovine serum; FMO: fluorescence-minusone; HSCT: hematopoietic stem cell transplantation; IFN- -1 -1 b: interferon-gamma1b; ISCT: International Society for Cellular Therapy; LL-CBMSC: long-living cord blood mesenchymal stromal cells; mAb: monoclonal antibody; MNC: mononuclear cells; MSC: mesenchymal stromal cells; PBMC: peripheral blood mononuclear cells; PBS: phosphate-buffered saline; GRT-PCR: quantitative real-time polymerase chain reaction; rh-IL2: recombinant human interleukin-2; rMFI: relative median fluorescence intensity; SL-CBMSC: short-living cord blood mesenchymal stromal cells; TNC: total nucleated cells; TNF-a: tumor necrosis factor alpha; UC: umbilical cord; USSC: unrestricted somatic stromal cells

\section{Acknowledgements}

We are grateful to all the mothers who gave their consent to donation. We are indebted to Anna Montaldi and Paola Celli for their important intellectual contribution to genetic data discussion. We really appreciated the contribution of Cinzia Tagliaferri and Monica Mantia to cord blood collection. We would like to thank Emanuele SG D'Amore and all the technicians of the Anatomy and Histology Department of S. Bortolo Hospital (Vicenza, Italy) for their technical assistance during the staining procedures. We are grateful to Paul Takam Kamga for critical review of the manuscript and to Carmine Carbone for his valuable contribution to molecular data analysis.

\section{Funding}

This work was partially supported by grants obtained from Cariverona Foundation (Research Project 2012.0828) and from Ricerca Sanitaria Finalizzata Regionale del Veneto 2012 (Research Project 334/12), assigned to Prof. Mauro Krampera, University of Verona (Italy).

\section{Authors' contributions}

EA and SS carried out the experimental design and analyzed/interpreted results. EA designed the research and wrote the manuscript. OP was involved in data interpretation, figure preparation, manuscript drafting and critical revisions for important intellectual content. MB, KC and DP provided technical assistance and participated in sample processing. $\mathrm{CL}$ was involved in sample collection and provided clinical parameters of CB donors. AZ performed molecular karyotyping and analysis of Additional file 8: Figure S5. AA, MR, FR and MR critically reviewed the manuscript. GA was involved in conception and design, coordination of the study, critical revisions for important intellectual content and manuscript final approval. All authors read and approved the manuscript for publication.

\section{Competing interests}

The authors declare that they have no competing interests.

\section{Consent for publication}

Informed consent included the consent for publication.

\section{Ethics approval and consent to participate}

The present study was approved (reference protocol SIT-VR 13/73) and informed consent to cord blood collection and donation for research was received from the mothers (specific section in the form IBMDR SCO101, version 2, 1-3 Jan 2013).

\section{Author details}

${ }^{1}$ Advanced Cellular Therapy Laboratory - Hematology Unit, S. Bortolo Hospital - ULSS 6, Contra' San Francesco 41, 36100 Vicenza, Italy. ${ }^{2}$ Transfusion Medicine, S. Bortolo Hospital, Vicenza, Italy. ${ }^{3}$ Hematology Project Foundation, Vicenza, Italy. ${ }^{4}$ Genetics and Molecular Biology, Transfusion Medicine, S. Bortolo Hospital, Vicenza, Italy.

Received: 4 September 2016 Revised: 13 December 2016 Accepted: 21 December 2016 Published online: 24 January 2017

\section{References}

1. Dominici M, et al. Minimal criteria for defining multipotent mesenchymal stromal cells. The International Society for Cellular Therapy position statement. Cytotherapy. 2006;8(4):315-7.

2. Aggarwal S, Pittenger MF. Human mesenchymal stem cells modulate allogeneic immune cell responses. Blood. 2005;105(4):1815-22.

3. Uccelli A, Moretta L, Pistoia V. Immunoregulatory function of mesenchymal stem cells. Eur J Immunol. 2006;36(10):2566-73. 
4. Nauta AJ, Fibbe WE. Immunomodulatory properties of mesenchymal stromal cells. Blood. 2007;110(10):3499-506.

5. Krampera M. Mesenchymal stromal cell 'licensing': a multistep process. Leukemia. 2011;25(9):1408-14.

6. Krampera M. Mesenchymal stromal cells: more than inhibitory cells. Leukemia. 2011;25(4):565-6.

7. Haniffa MA, et al. Mesenchymal stem cells: the fibroblasts' new clothes? Haematologica. 2009;94(2):258-63.

8. Ball LM, et al. Cotransplantation of ex vivo expanded mesenchymal stem cells accelerates lymphocyte recovery and may reduce the risk of graft failure in haploidentical hematopoietic stem-cell transplantation. Blood. 2007;110(7):2764-7.

9. de Lima $\mathrm{M}$, et al. Cord-blood engraftment with ex vivo mesenchymal-cell coculture. N Engl J Med. 2012;367(24):2305-15.

10. Sanina C, Hare JM. Mesenchymal stem cells as a biological drug for heart disease: where are we with cardiac cell-based therapy? Circ Res. 2015;117(3): 229-33.

11. Le Blanc K, et al. Treatment of severe acute graft-versus-host disease with third party haploidentical mesenchymal stem cells. Lancet. 2004;363(9419): 1439-41.

12. Introna M, Rambaldi A. Mesenchymal stromal cells for prevention and treatment of graft-versus-host disease: successes and hurdles. Curr Opin Organ Transplant. 2015;20(1):72-8.

13. Pittenger MF, et al. Multilineage potential of adult human mesenchymal stem cells. Science. 1999;284(5411):143-7.

14. Zuk PA, et al. Human adipose tissue is a source of multipotent stem cells. Mol Biol Cell. 2002;13(12):4279-95.

15. Erices $A$, Conget $P$, Minguell JJ. Mesenchymal progenitor cells in human umbilical cord blood. Br J Haematol. 2000;109(1):235-42.

16. Montesinos JJ, et al. Human mesenchymal stromal cells from adult and neonatal sources: comparative analysis of their morphology, immunophenotype, differentiation patterns and neural protein expression. Cytotherapy. 2009;11(2):163-76.

17. Reinisch A, et al. Humanized system to propagate cord blood-derived multipotent mesenchymal stromal cells for clinical application. Regen Med. 2007;2(4):371-82.

18. Kern $\mathrm{S}$, et al. Comparative analysis of mesenchymal stem cells from bone marrow, umbilical cord blood, or adipose tissue. Stem Cells. 2006;24(5): 1294-301.

19. Avanzini MA, et al. Generation of mesenchymal stromal cells in the presence of platelet lysate: a phenotypic and functional comparison of umbilical cord blood- and bone marrow-derived progenitors. Haematol Hematol J. 2009;94(12):1649-60.

20. Zhang $X$, et al. Isolation and Characterization of mesenchymal stem cells from human umbilical cord blood: reevaluation of critical factors for successful isolation and high ability to proliferate and differentiate to chondrocytes as compared to mesenchymal stem cells from bone narrow and adipose tissue. J Cell Biochem. 2011;112(4):1206-18.

21. Barilani $\mathrm{M}$, et al. Dissection of the cord blood stromal component reveals predictive parameters for culture outcome. Stem Cells Dev. 2015;24(1):104-14.

22. Castro-Manrreza ME, et al. Human mesenchymal stromal cells from adult and neonatal sources: a comparative in vitro analysis of their immunosuppressive properties against T cells. Stem Cells Dev. 2014;23(11): 1217-32.

23. Cutler AJ, et al. Umbilical cord-derived mesenchymal stromal cells modulate monocyte function to suppress T cell proliferation. J Immunol. 2010;185(11): 6617-23.

24. van den Berk LC, et al. Cord blood mesenchymal stem cells suppress DC-T Cell proliferation via prostaglandin B2. Stem Cells Dev. 2014;23(14):1582-93.

25. Wang $M$, et al. The immunomodulatory activity of human umbilical cord blood-derived mesenchymal stem cells in vitro. Immunology. 2009;126(2): 220-32.

26. Lee $\mathrm{SH}$, et al. Co-transplantation of third-party umbilical cord blood-derived MSCs promotes engraftment in children undergoing unrelated umbilical cord blood transplantation. Bone Marrow Transplant. 2013;48(8):1040-5.

27. Kim HS, et al. Clinical trial of human umbilical cord blood-derived stem cells for the treatment of moderate-to-severe atopic dermatitis: phase I/lla studies. Stem Cells. 2017;35(1):248-55.

28. Astori $\mathrm{G}$, et al. Cord blood-derived mesenchymal stromal cells for the treatment of graft-versus host disease following hematological stem cell transplantation. Cytotherapy. 2016;18(6):S19.
29. Bieback K, et al. Critical parameters for the isolation of mesenchymal stem cells from umbilical cord blood. Stem Cells. 2004;22(4):625-34.

30. Sibov TT, et al. Mesenchymal stem cells from umbilical cord blood: parameters for isolation, characterization and adipogenic differentiation. Cytotechnology. 2012;64(5):511-21.

31. Vasaghi $A$, et al. Parameters that influence the isolation of multipotent mesenchymal stromal cells from human umbilical cord blood. Hematol Oncol Stem Cell Ther. 2013;6(1):1-8.

32. Barilani $M$, et al. A chemically defined medium-based strategy to efficiently generate clinically relevant cord blood mesenchymal stromal colonies. Cell Transplant. 2016;25(8):1501-14.

33. Laitinen $\mathrm{A}$, et al. The effects of culture conditions on the functionality of efficiently obtained mesenchymal stromal cells from human cord blood. Cytotherapy. 2016;18(3):423-37.

34. Kögler $\mathrm{G}$, et al. A new human somatic stem cell from placental cord blood with intrinsic pluripotent differentiation potential. J Exp Med. 2004;200(2): 123-35.

35. Pievani $\mathrm{A}$, et al. Comparative analysis of multilineage properties of mesenchymal stromal cells derived from fetal sources shows an advantage of mesenchymal stromal cells isolated from cord blood in chondrogenic differentiation potential. Cytotherapy. 2014;16(7):893-905.

36. Ragni $E$, et al. What is beyond a qRT-PCR study on mesenchymal stem cell differentiation properties: how to choose the most reliable housekeeping genes. J Cell Mol Med. 2013;17(1):168-80.

37. Krampera $\mathrm{M}$, et al. Immunological characterization of multipotent mesenchymal stromal cells-The International Society for Cellular Therapy (ISCT) working proposal. Cytotherapy. 2013;15(9):1054-61.

38. Samsonraj RM, et al. Establishing criteria for human mesenchymal stem cell potency. Stem Cells. 2015;33(6):1878-91.

39. Corselli $\mathrm{M}$, et al. Identification of perivascular mesenchymal stromal/stem cells by flow cytometry. Cytometry A. 2013;83(8):714-20.

40. Bassi $\mathrm{G}$, et al. Effects of a ceramic biomaterial on immune modulatory properties and differentiation potential of human mesenchymal stromal cells of different origin. Tissue Eng Part A. 2015;21(3-4):767-81.

41. Galipeau J, Krampera M. The challenge of defining mesenchymal stromal cell potency assays and their potential use as release criteria. Cytotherapy. 2015;17(2):125-7.

42. Galipeau J, et al. International Society for Cellular Therapy perspective on immune functional assays for mesenchymal stromal cells as potency release criterion for advanced phase clinical trials. Cytotherapy. 2016;18(2):151-9.

43. Zeddou M, et al. The umbilical cord matrix is a better source of mesenchymal stem cells (MSC) than the umbilical cord blood. Cell Biol Int 2010;34(7):693-701.

44. Wang $\mathrm{H}$, et al. Dexamethasone has variable effects on mesenchymal stromal cells. Cytotherapy. 2012;14(4):423-30.

45. Capelli C, et al. Minimally manipulated whole human umbilical cord is a rich source of clinical-grade human mesenchymal stromal cells expanded in human platelet lysate. Cytotherapy. 2011;13(7):786-801.

46. Sensebé $L$, et al. Limited acquisition of chromosomal aberrations in human adult mesenchymal stromal cells. Cell Stem Cell. 2012;10(1):9-10. author reply 10-1.

47. Sensebé L. Beyond genetic stability of mesenchymal stromal cells Cytotherapy. 2013;15(11):1307-8.

48. Ragni $E$, et al. Adipogenic potential in human mesenchymal stem cells strictly depends on adult or foetal tissue harvest. Int J Biochem Cell Biol. 2013;45(11):2456-66.

49. Karagianni $\mathrm{M}$, et al. A comparative analysis of the adipogenic potential in human mesenchymal stromal cells from cord blood and other sources. Cytotherapy. 2013;15(1):76-88.

50. Chang YJ, et al. Disparate mesenchyme-lineage tendencies in mesenchymal stem cells from human bone marrow and umbilical cord blood. Stem Cells. 2006;24(3):679-85.

51. Abdulrazzak $\mathrm{H}$, et al. Biological characteristics of stem cells from foetal, cord blood and extraembryonic tissues. J R Soc Interface. 2010;7 Suppl 6:5689-706

52. Crisan $\mathrm{M}$, et al. A perivascular origin for mesenchymal stem cells in multiple human organs. Cell Stem Cell. 2008;3(3):301-13.

53. Sacchetti $B$, et al. No identical "mesenchymal stem cells" at different times and sites: human committed progenitors of distinct origin and differentiation potential are incorporated as adventitial cells in microvessels. Stem Cell Rep. 2016;6(6):897-913. 
54. Kluth SM, et al. DLK-1 as a Marker to distinguish unrestricted somatic stem cells and mesenchymal stromal cells in cord blood. Stem Cells Dev. 2010;19(10):1471-83.

55. Kluth SM, Radke TF, Koegler G. Increased haematopoietic supportive function of USSC from umbilical cord blood compared to CB MSC and possible role of DLK-1. Stem Cells Int. 2013;2013:985285.

56. Liedtke $\mathrm{S}$, et al. Low oxygen tension reveals distinct HOX codes in human cord blood-derived stromal cells associated with specific endochondral ossification capacities in vitro and in vivo. J Tissue Eng Regen Med. 2016 doi:10.1002/term.2167. [Epub ahead of print] PubMed PMID: 27214005.

57. Menard C, et al. Clinical-grade mesenchymal stromal cells produced under various good manufacturing practice processes differ in their immunomodulatory properties: standardization of immune quality controls. Stem Cells Dev. 2013;22(12):1789-801.

58. Ketterl $\mathrm{N}$, et al. A robust potency assay highlights significant donor variation of human mesenchymal stem/progenitor cell immune modulatory capacity and extended radio-resistance. Stem Cell Res Ther. 2015;6:236.

59. Bloom DD, et al. A reproducible immunopotency assay to measure mesenchymal stromal cell-mediated T-cell suppression. Cytotherapy. 2015;17(2):140-51.

60. von Bahr $\mathrm{L}$, et al. Long-term complications, immunologic effects, and role of passage for outcome in mesenchymal stromal cell therapy. Biol Blood Marrow Transplant. 2012;18(4):557-64.

61. Schepers K, Fibbe WE. Unraveling mechanisms of mesenchymal stromal cell-mediated immunomodulation through patient monitoring and product characterization. Ann N Y Acad Sci. 2016;1370(1):15-23.

\section{Submit your next manuscript to BioMed Central and we will help you at every step:}

- We accept pre-submission inquiries

- Our selector tool helps you to find the most relevant journal

- We provide round the clock customer support

- Convenient online submission

- Thorough peer review

- Inclusion in PubMed and all major indexing services

- Maximum visibility for your research

Submit your manuscript at www.biomedcentral.com/submit 